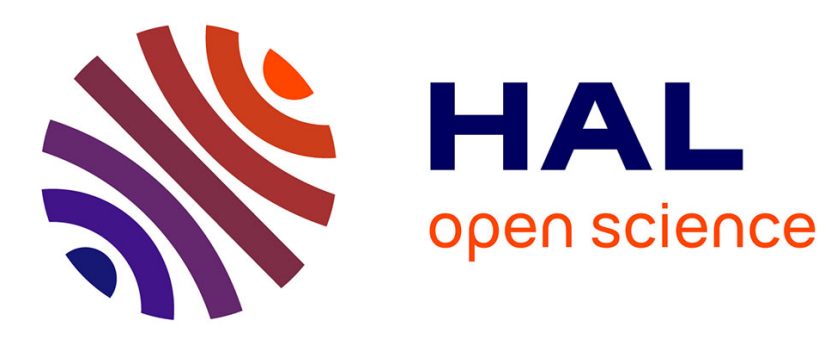

\title{
Bond-orientational order in Langmuir-Blodgett surfactant monolayers
}

S. Garoff, H.W. Deckman, J.H. Dunsmuir, M.S. Alvarez, J.M. Bloch

\section{To cite this version:}

S. Garoff, H.W. Deckman, J.H. Dunsmuir, M.S. Alvarez, J.M. Bloch. Bond-orientational order in Langmuir-Blodgett surfactant monolayers. Journal de Physique, 1986, 47 (4), pp.701-709. 10.1051/jphys:01986004704070100 . jpa-00210251

\section{HAL Id: jpa-00210251 https://hal.science/jpa-00210251}

Submitted on 1 Jan 1986

HAL is a multi-disciplinary open access archive for the deposit and dissemination of scientific research documents, whether they are published or not. The documents may come from teaching and research institutions in France or abroad, or from public or private research centers.
L'archive ouverte pluridisciplinaire HAL, est destinée au dépôt et à la diffusion de documents scientifiques de niveau recherche, publiés ou non, émanant des établissements d'enseignement et de recherche français ou étrangers, des laboratoires publics ou privés. 
Classification

Physics Abstracts

$68.60-61.14 \mathrm{~F}-61.30 \mathrm{E}$

\title{
Bond-orientational order in Langmuir-Blodgett surfactant monolayers
}

\author{
S. Garoff, H. W. Deckman, J. H. Dunsmuir, M. S. Alvarez \\ Exxon Research and Engineering Company, Route 22 East, Annandale, New Jersey 08801, U.S.A.
}

and J. M. Bloch

Brookhaven National Laboratory, Upton, NY 11973, U.S.A.

(Reçu le 22 octobre 1985, accepté le 5 décembre 1985)

\begin{abstract}
Résumé. - Des monocouches de stéarate de cadmium ont été préparées selon la méthode de Langmuir-Blodgett. Un ordre orientationnel des liaisons des molécules de tensioactif a été mis en évidence. Les corrélations orientationnelles des liaisons s'étendent sur des distances macroscopiques $(\approx 1 \mathrm{~mm})$, alors que les corrélations translationnelles décroissent sur une longueur de dizaines d'angstroms. L'axe des molécules est incliné par rapport à la normale à la surface mais la direction de l'inclinaison est aléatoire. Nos mesures de diffraction électronique suggèrent une agrégation des chaînes aliphatiques au sein de la monocouche.
\end{abstract}

\begin{abstract}
Cadmium stearate surfactant molecules assembled into monolayers by the Langmuir-Blodgett method exhibit bond-orientational order. Bond-orientational correlations extend over macroscopic distances on the order of a millimeter while translational correlations decay on a scale of tens of angstroms. The long axis of the molecules are tilted away from the surface normal but the tilt direction is disordered. Our results, obtained by transmission electron diffraction, suggest clustering of the hydrocarbon chains in the monolayer.
\end{abstract}

\section{Introduction.}

Surface bound monolayers of amphiphilic molecules, commonly known as surfactants, can dramatically alter such interfacial properties as wetting of a solid by a fluid [1] or lubrication of the motion of two contacting solid surfaces [2]. Bilayers of certain amphiphilic molecules are thought to mimic some properties of biological membranes. Recently, multilayers have been proposed as components of electronic and optical devices [3]. The Langmuir-Blodgett (LB) method of " pre-assembling " a molecular layer at the air/water interface and transferring one monolayer at a time to a solid substrate is one of the most popular methods of preparing such layered systems. Structural studies of LB films are essential to understanding the fundamental properties of layered assemblies of surfactant molecules. In particular, structural analysis of the molecular ordering within a single LB monolayer is important both to understand how the environment in the immediate vicinity of the surface affects the structure of the molecular monolayer and to ascertain how the structure of one layer forms a template for subsequent layers in a multilayer formation. In this paper, we discuss a detailed analysis of the structure of an LB monolayer using transmission electron diffraction techniques.

Studies of the order within surfactant monolayers have been performed for many years. Multilayer assemblies have been studied by electron $[4,5]$ and $\mathrm{X}$-ray diffraction [6] as well as infrared absorption $[5,7,8]$. Associated with probes of lubrication by single surfactant monolayers, reflection electron diffraction has been used to examine a variety of $L B$ and solution adsorbed surfactant systems [2]. In addition, transmission electron diffraction studies were performed on monolayers of fatty acids and their salts $[5,9$, $10]$, and lipids [11, 12]. To date, only one X-ray study of in-plane ordering in a monolayer has been published [13]. Previous diffraction studies have determined lattice constants and symmetries of the single monolayer. In this work, we quantitatively analyse the structure of an LB monolayer of cadmium stearate using electron diffraction, which allows us to rapidly probe all relevant parts of reciprocal space including scans along the Bragg rods produced by the monolayers. Because of the small scattering strength of the monolayer, kinematic or single scattering theory can be used to derive a structural picture of the monolayer from the diffraction results. 
The LB monolayers prepared for this study exhibit long-range bond-orientational correlations but poor translational order. The long alkyl chains of the molecules tilt away from the surface normal with the tilt direction azimuthally disordered. We discuss this structure in terms of molecular models which reproduce the fundamental trends of the diffraction pattern. Our results suggest clustering of the hydrocarbon chains in the monolayer and raise questions concerning the effects of preparative details and the substrate on the monolayer structure. We also find that the molecular stoichiometry of the monolayer is different from that found in multilayer assemblies. In the next section, we discuss our experimental methods for preparing the films and for collecting and processing the electron diffraction data. Section 3 contains the details of our results and of the structural picture we obtain. The final section provides a summary and further comments on the implications of our results both for the microscopic forces within the monolayer as well as for their effect on macroscopic interfacial behaviour.

\section{Experimental procedures.}

The monolayers are made by classic LangmuirBlodgett procedures. All materials used are of the highest possible purity and cleanliness is maintained at each stage of sample preparation. The subphase contains $10^{-4} \mathrm{M} \mathrm{CdCl}_{2}$ (Puratronic grade from Alfa Products) and has a $\mathrm{pH}=6.8$, adjusted using $\mathrm{HCl}$ and $\mathrm{NH}_{4} \mathrm{OH}$ (both ultrapure grade from Alfa Products). Stearic acid $(99.999 \%$ pure from Altech Assoc.) is applied to the air/water interface from a spreading solution of chloroform (ultrapure grade from Alfa Products). Using a solution containing $100 \mathrm{mg}$ of acid/100 ml of solution, the surfactant is put on the surface to an initial concentration of roughly $50 \AA^{2}$ / molecule. Then, the surface pressure is allowed to equilibrate as the solvent evaporates from the surface (this takes about $20 \mathrm{~min}$ ). The film is compressed at $0.01 \AA / \mathrm{molecule} / \mathrm{s}$ to a surface pressure of about 10 dynes/cm, just above the break between the « liquidcondensed " and solid phases [14] in the pressure/area isotherm. With surface pressure fixed, the film is allowed to relax from the initial area per stearic acid molecule of $25 \AA^{2}$ to its asymptotic value of 21 to $23 \AA^{2}$. Relaxation takes about $30 \mathrm{~min}$.

The substrates are $20 \AA$ thick layers of $\mathrm{SiO}$ coated on $100 \AA$ thick layers of stress free amorphous carbon on 200 mesh Ni electron microscopy grids. Each grid is mounted in a milled indentation on a silicon wafer so that the top surface of the grid is flush with the wafer surface. Thus during film deposition, the meniscus passes undeformed past the grid. Flatness of the substrate is examined both before and after monolayer deposition by optical interferometry and by shadowing electron microscopy methods. The surface is macroscopically flat; and by inference from the regions shadowed during low angle deposition of erbium, the surface shows microstructural features less than $10 \AA$ high and larger than $600 \AA$ in lateral dimensions implying maximum surface inclinations of $1^{\circ}$ to $3^{\circ}$ from the average surface normal direction. The substrate is pulled through the air/water interface at $50 \mu \mathrm{m} / \mathrm{s}$ and the resulting film has a transfer ratio within $5 \%$ of unity. The films are stable for weeks in ambient conditions and more than 3 days in the vacuum of the electron microscope.

The electron diffraction methods used in this study provide many benefits for probing the ordering in surfactant monolayers. Because the wavelength of the electrons $(\lambda=0.037 \AA)$ is much smaller than the lattice constant of the structure, the Ewald sphere is essentially flat over the region of reciprocal space in which diffracted intensity lies. Thus, with one exposure of the photographic film in the detection plane, intensity distributions in all Bragg scattering rods are simultaneously recorded. This rapid data acquisition has allowed us to examine more than 500 cross sectional views of Bragg rod from over 100 locations on several monolayer samples. In addition, use of the electron microscope allows us to image the areas of the film to be examined by diffraction. Therefore, we can ensure that these areas are representative of the whole film and are not near microscopic defects in the monolayer or the substrate. Since the total diffracted intensity from a sample is less than $1 \%$ of the incident beam, the scattering from the monolayer can be assumed to be in the single scattering or kinematic limit. Analysis of diffraction patterns need not account for multiple scattering as is often the case in electron diffraction studies. Finally, as will be explained later, the electron beam does not alter the monolayer structure during the recording of the diffraction pattern.

The diffraction experiments are performed on a Phillips $400 \mathrm{~T}$ transmission electron microscope. Illumination conditions of the microscope are set up to minimize beam currents at the sample ( 0.02 electron $/ \AA^{2}$ s) and to produce parallel illumination of the sample by the incident beam of $100 \mathrm{keV}$ electrons. The photographic film (Kodak 4489) and developing conditions (Kodak HRP, $4 \mathrm{~min}$ ) record the diffraction patterns with high resolution and short exposures. For these conditions, the sensometric curve is linear over 4 units of optical density (OD), with

$\mathrm{OD} \alpha\left(5.1 \times 10^{10}\right) e$

$$
5 \times 10^{-12} \leqslant e \leqslant 100 \times 10^{-12}
$$

where $e$ is the electron exposure $\left(\mathrm{C} / \mathrm{cm}^{2}\right)$ at the photographic film surface. Linearity of the sensometric curve significantly simplifies quantitative interpretation of recorded diffraction patterns. For these illumination conditions and film response, a diffraction pattern can be recorded from a $125 \mu \mathrm{m}^{2}$ area in $10 \mathrm{~s}$. However, visual observations can be made on the microscope phosphor screen in less than $1 \mathrm{~s}$. Alternately the higher sensitivity of the visual observations allows 
the structure of areas as small as $1 \mu \mathrm{m}^{2}$ to be qualitatively probed. These short cumulative exposures are more than an order of magnitude lower than electron beam damage thresholds for similar LB films of lipids [15]. Lattice constant measurements are directly calibrated using aluminum films deposited on the same grid as the LB film. To probe diffracted intensity along Bragg rods perpendicular to the monolayer plane, the sample is tilted relative to the incident beam. The rotation of the diffraction pattern relative to the specimen tilt axis due to the electron optics is uniquely determined from the diffraction of an $\mathrm{MoO}_{3}$ crystallite which has a highly anisotropic growth habit. Thus the orientation of the Bragg rods can be referred directly back to the specimen tilt axis. Diffracted intensity data is obtained by scanning the photographic plates with a Joyce-Lobel scanning microdensitometer.

The resolution limits and accuracy of our measurements are a function of the electron optics, the photographic plates, and the densitometer used to measure the patterns from the plates. We determine the resolution function experimentally by measuring the diffraction pattern from a very small $(\approx 2 \mu \mathrm{m} \times$ $2 \mu \mathrm{m} \times 200 \AA)$ crystallite of cadmium stearate. From this measurement, we find that the resolution limited linewidth $\left(\approx 0.002 \AA^{-1}\right)$ is about $1 / 20$ the linewidth of the narrowest diffraction profile from the monolayer. Because of mechanical motions of the microdensitometer, difficulties in measuring the centres of diffraction features, as well as small errors in focussing of the electron optics, spacings between features are only measured to about $5 \%$ accuracy.

\section{Results and discussions.}

A typical diffraction pattern is shown in figure 1 with coordinates used in the subsequent discussion. The pattern shows a hexagonal array of 6 equally spaced, diffuse $\langle 100\rangle$ Bragg spots in the $q_{z}=0$ plane. Pairs of diametrically opposing spots must be of equal intensity because of the inversion symmetry of diffraction patterns in the kinematic limit. Measured intensities of spot pairs from many different patterns show $6 \%$ differences and this identifies our experimental error in measuring the peak intensities. In fact, all $6<100>$ spots on any pattern fell within these error limits and thus our diffraction pattern indicates a fully hexagonally symmetric structure. The $\langle 100\rangle d$-spacing is $4.20 \pm 0.10 \AA$, indicating a spacing between alkyl chains of $4.89 \AA$. This symmetry and packing density is similar to that found for chains of lipid monolayers [16] and in other fatty acid monolayers [5, 17]. Further, this $d$-spacing indicates an area/chain of $21 \AA^{2}$ in good agreement with the area/chain in the precursing film on the trough. The $\langle 110\rangle d$-spacing is $1 / \sqrt{3}$ of the $\langle 100\rangle$ spacing as it should be for a hexagonal structure.

Although at the subphase $\mathrm{pH}$ used in our deposition, bulk analysis shows that one cadmium ion is coor-

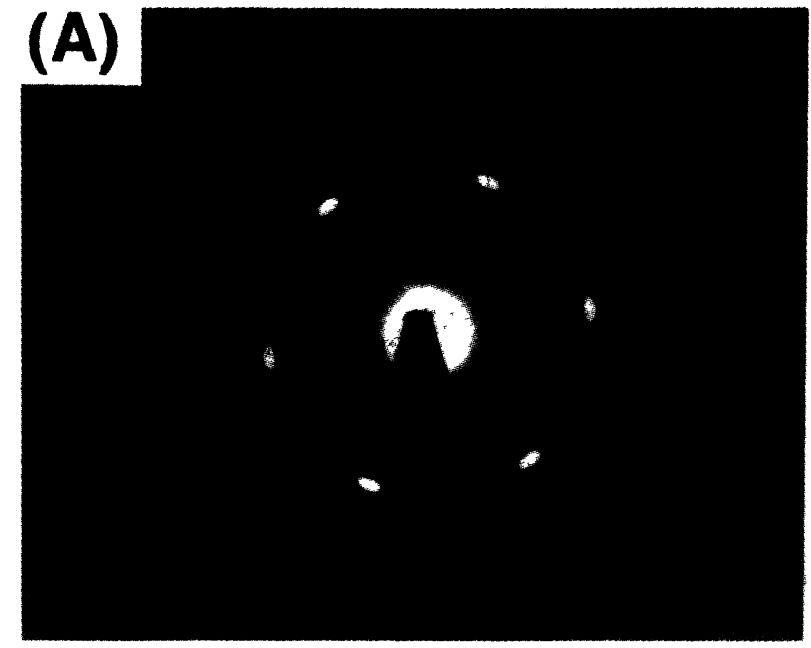

\section{(B)}
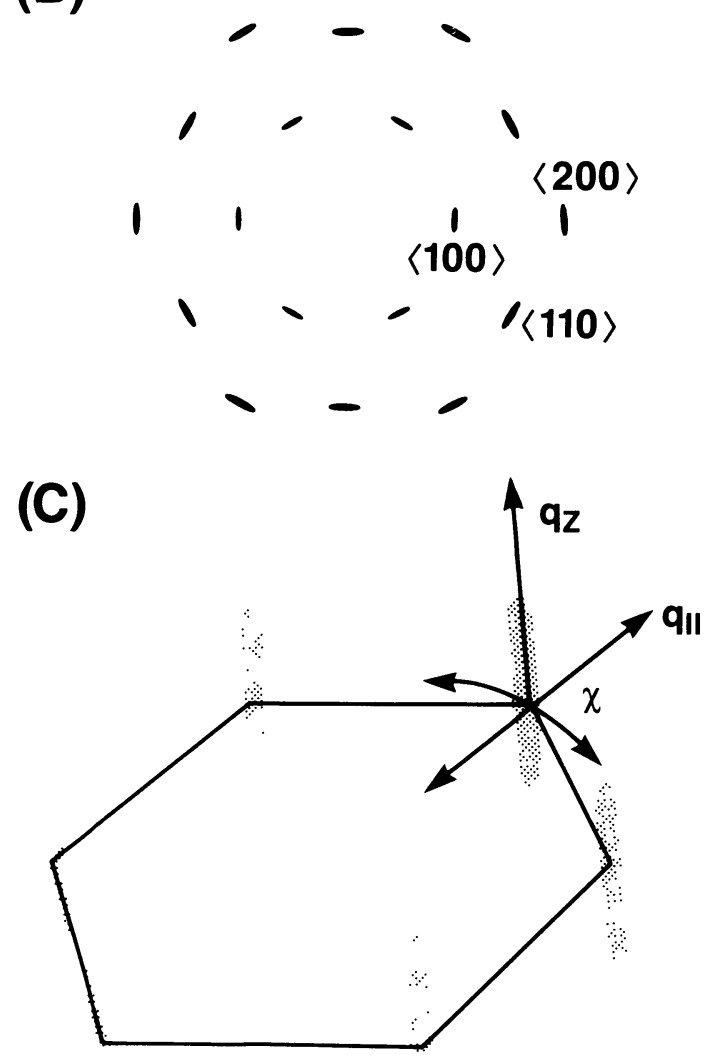

Fig. 1. - (a) Typical diffraction pattern for $q_{z}=0$. Higher order spots are not visible because of their low intensity. (b) Indexing of diffraction spots. (c) Coordinates of reciprocal space.

dinated to 2 stearate ions in multilayer films [18], our diffraction pattern indicates this is not true in the first monolayer. The small $d$-spacing of the $\langle 100\rangle$ spots indicates the unit cell is too small to contain more than one stearate ion. Cadmiums shared by two stearate ions could not also be on a hexagonal lattice. Although the atomic scattering factor of cadmium is 
$\approx 4$ times that of a carbon atom [19], we have seen no indication of superlattice spots. Further, if the cadmium did join two stearate ions, some distortion of the hexagonal symmetry of the stearate net should occur. No such distortion is observed. The association of a single alkanoate ion with a divalent cation has been observed for a calcium behenate monolayer deposition on alumina [20]. Auger analysis of cadmium stearate LB monolayers prepared on gold substrates identified chloride as the second counterion associated with the cadmium [21]. Thus, the chemical composition of the first layer on the substrate is different than that observed in multilayers and the cadmium appears to sit on the same hexagonal net as the stearate ions.

The density and symmetry of the lattice also indicates the conformation and rotational state of the hydrocarbon chain of the stearate ion. The dense packing of the structure is only slightly larger than the hard core size of an alkyl chain. Theoretical calculations predict that at the densities present in this monolayer few or no gauche conformations would be present in the chains [22]. Further, the hexagonal symmetry of the structure shows that the stearate ions are in a rotator phase [16, 23], i.e. a state where the chains have considerable rotational freedom about their long axis and the plane of the zigzag configuration of the carbon backbone of one chain is not locked into a specific orientation relative to that of neighbouring chains.

Translational disorder is indicated by many aspects of the diffraction pattern. Scans of diffracted intensity along a radial direction (along $q_{\|}$) through the centre of $\langle 100\rangle$ Bragg spots show a broad distribution of intensity with a full width at half maximum (FWHM) of $0.05 \AA^{-1}$, corresponding to a correlation length of $40 \AA$. Photographs of diffraction patterns of other LB monolayers qualitatively show poor translational order [5, 9, 12]. A typical radial scan is shown in figure 2 . Using standard least squares methods, the fit of these radial scans to a Lorentzian profile produces a reduced chi-square four times smaller than that for a Gaussian fit. As seen in figure 2, the tails of the peak clearly show the slow (roughly $\Delta q^{-2}$ ) fall-off of the Lorentzian lineshape characteristic of liquid-like translational correlations. The larger FWHM of the $\langle 110\rangle$ Bragg spot (4 times the FWHM of the $\langle 100\rangle$ spot) and lower intensities of the $\langle 110\rangle$ and $\langle 200\rangle$ spots ( 0.01 to 0.05 of the $\langle 100\rangle$ intensity) are also characteristic of positional disorder in the structure. The FWHM of the radial scan varies with azimuthal angle $(\chi)$ giving intensity contours of the $\langle 100\rangle$ spot an oval shape. This spot shape will be shown to be indicative of the type of translational disorder. Finally, radial scans at higher positions along the Bragg rods (i.e. at increasing values of $q_{z}$ ) show increasing FWHM. At $q_{z}=0.02 \AA^{-1}$ (where the peak intensity is roughly $1 / 2$ of the intensity at $q_{z}=0$ ), the FWHM of the radial scan has increased by about $20 \%$ over the value at

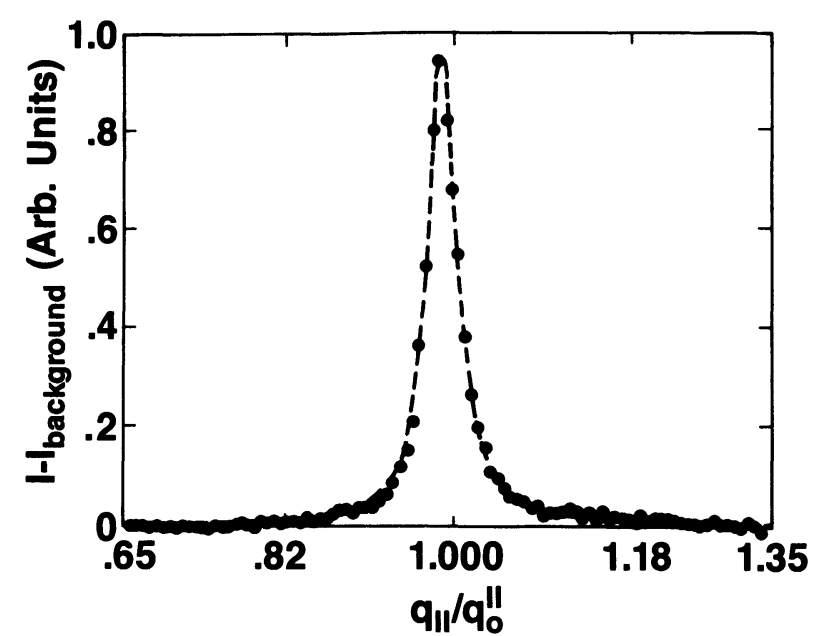

Fig. 2. - Radial scan through Bragg spot at $\chi=0$ and $q_{z}=0$. (...) Data; (---) best fit Lorentzian lineshape. $q_{0}^{\|}=1.496 \AA^{-1}(=2 \pi /$ lattice constant $)$.

$q_{z}=0$; and at $q_{z}=0.26 \AA^{-1}$ (where the peak intensity is $1 / 3$ of the intensity at $q_{z}=0$ ), the FWHM of the radial scan is about 2 times the value at $q_{z}=0$. No change in $d$-space is detected as $q_{z}$ increases. This increasing linewidth with $q_{z}$ provides evidence of a dependence of correlations between atoms on position along the layer normal.

The poor degree of translational order is in strong contrast to the high degree of bond orientational order found in the monolayer. The angular distribution of diffracted intensities (along the $\chi$ direction) indicates that the monolayer has a very high correlation of bond orientations. These azimuthal scans through the $\langle 100\rangle$ Bragg spots show a FWHM of about $8^{\circ}$ (see Fig. 3), and the ratio of intensities $30^{\circ}$ from the peak to that in the peak is typically 0.05 . Strikingly, the orientation of the 6-fold pattern does not vary more than $\approx 5^{\circ}$ as the sample is translated over macroscopic distances $(\approx 1 \mathrm{~mm})$. Thus, the bond

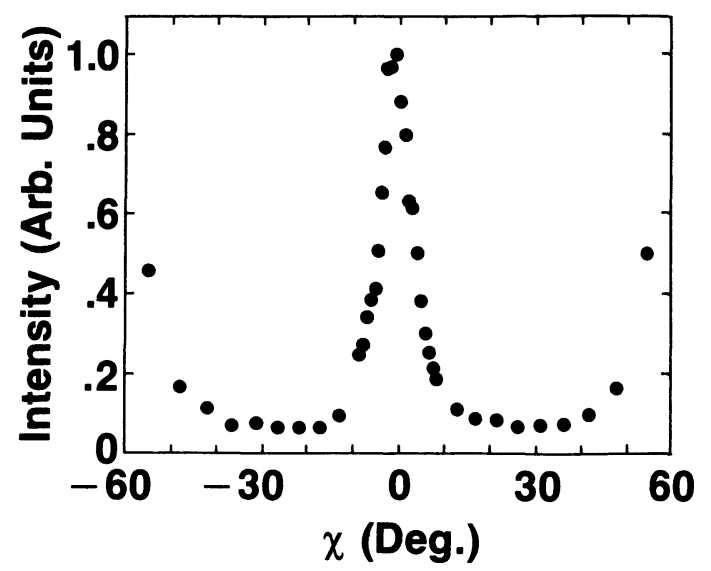

Fig. 3. $-\chi$ scan through Bragg spot at $q_{\|}=q_{0}^{\|}=1.496 \AA^{-1}$ and $q_{z}=0$. 
orientations of the film are highly correlated across greater than $10^{6}$ unit cells !

Since the molecules composing the film are rod-like entities, disorder in the structure may arise from three sources : a) positional disorder of the molecules with either liquid-like or microcrystalline correlations; b) disorder in the magnitude of the tilting of the rodlike molecules relative to the surface normal; and c) disorder in the direction of this tilt. One can easily see that b) and c) can contribute to degradation of translational correlations in a structure because they impose a distribution of distances between the atoms of two neighbouring chains.

A semiquantitative separation of these three separate aspects of disorder is obtained using calculated diffraction patterns from simplified arrangements of rod-like molecules to probe how each type of disorder contributes to trends in symmetry, linewidths and intensities. Because of the low $z$ dependence for the atomic cross sections for electron diffraction, structures made up of only a simple eighteen carbon chain show virtually the same diffraction pattern as those made up of a more accurate representation of the cadmium stearate containing both cadmium and oxygen atoms. Thus, we neglect the presence of the heteroatoms in our modelling. Based on the observed dense packing and hexagonal symmetry of the monolayer, we further assume that the building block of the structure is a planar zigzag arrangement of carbon atoms with free rotation of the planar structure about the long axis of the molecule. Using the positions of carbons in a simple alkane chain [24], we derive the form factor for the unit cell of a single representative carbon chain

$$
\begin{aligned}
|F|^{2} \alpha \frac{J_{1}^{2}(R|q|)}{|q|^{2}}\left\{1+\left[\cos \left(1.25 q_{z}\right)\right] J_{0}\left(0.89 \sqrt{q_{x}^{2}+q_{y}^{2}}\right)\right\} \frac{\sin ^{2} \frac{45}{4} q_{z}}{\sin ^{2} \frac{5}{4} q_{z}} \\
q_{x}=\tilde{q}_{x} \cos \alpha+\widetilde{q}_{y} \sin \alpha \\
q_{y}=-\widetilde{q}_{x} \cos \theta \sin \alpha+\widetilde{q}_{y} \cos \theta \cos \alpha+\tilde{q}_{z} \sin \theta \\
q_{z}=\tilde{q}_{x} \sin \theta \sin \alpha-\widetilde{q}_{y} \sin \theta \cos \alpha+\widetilde{q}_{z} \cos \theta
\end{aligned}
$$

where $\tilde{q}_{i}$ are the coordinates of the $\widetilde{\mathbf{q}}$ in the laboratory frame, $\theta$ is the magnitude of the tilt angle of the rod relative to the surface normal, $\alpha$ is the direction of the tilt relative to the $\tilde{q}_{x}$ direction, $R$ is the effective radius of the carbon atom, and $J_{0}$ and $J_{1}$ are Bessel functions. The radial direction, $q_{\|}$, is $\left(q_{x}^{2}+q_{y}^{2}\right)^{1 / 2}$. To mimic positional disorder we assume the molecules are arranged into microcrystallites within which the positional correlations are perfect. The truncation of the crystallite imposes a form of disorder on the total structure which is similar to other positional disorder mechanisms. To maintain the bond oriented features in our data, we incoherently superimpose the diffraction from these crystallites but assume the bonds of these independent crystallites are aligned. By assigning a tilt magnitude and direction to each molecule in the microcrystal, we can simulate the effects of tilting of the molecules and even disorder in the tilts.

Scans of the diffraction along the Bragg rods (along $q_{z}$ ) provide information about the tilt magnitude and direction of the molecules. The diffracted intensity along the rod is only slightly affected by inplane ordering; and to first order, the width of the $q_{z}$ scan gives the effective thickness of the layer. For monolayers of tilted molecules, the width of the $q_{z}$ lineshape is increased because of the decrease in the thickness of the layer. In addition, the maximum of the form factor of the tilted molecule is pushed out of the $q_{z}=0$ plane for $q_{\|} \neq 0$. The linewidth for the profile of diffracted intensity along $q_{z}$ (see Fig. 4) can be simulated by a layer with a dominant tilt angle of about $8^{\circ}$. To emphasize the broader lineshape observed for the monolayer compared to that expected for a layer of untilted molecules, we have plotted the calculated diffraction from an untilted layer in figure 4 [25]. However, if the molecules are all tilted in the same

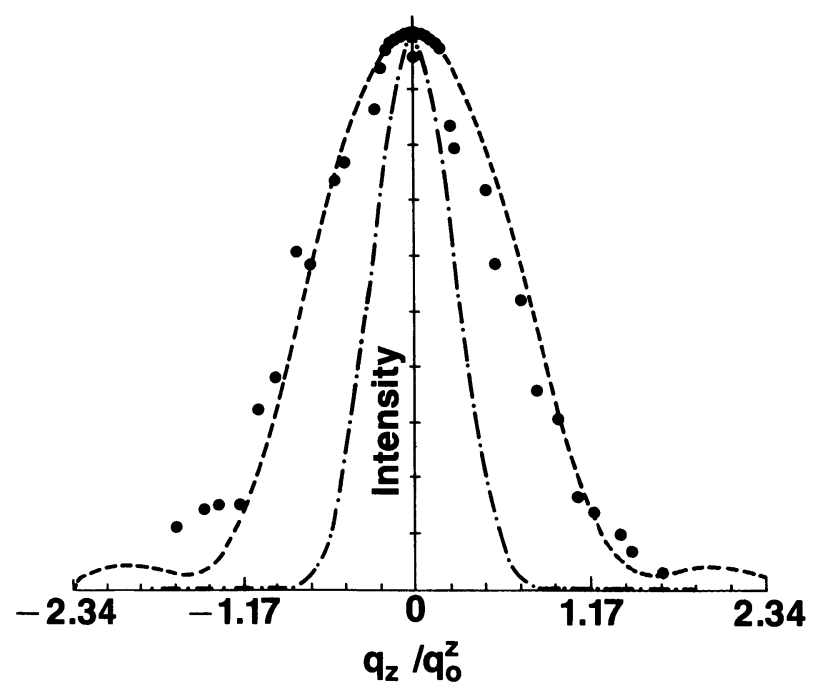

Fig. 4. $-q_{z}$ scan along Bragg rod at $\chi=0$ and $q_{\|}=q_{0}^{\|}=$ $1.496 \AA^{-1} . \quad q_{0}^{z}=0.269 \AA^{-1}(2 \pi /$ length of hydrocarbon chain). (....) Data; (---) model for tilted molecules ; (.-.-.-...) model for untilted molecules. 
direction, then the intensities of the 3 pairs of $\langle 100\rangle$ spots in the $q_{z}=0$ plane would be dramatically different even for small tilts. Thus, the observed equality of the intensities of all six spots indicates an averaging of the tilt direction within the sampling area, i.e. down to $1 \mu \mathrm{m}^{2}$. Further, this averaging causes the diffraction pattern to have mirror symmetry about the $q_{z}=0$ plane even though the form factors for the individual molecules do not. Within our detectability limits, there is no distortion of the hexagonal lattice due to this molecular tilt. This contrasts with smectic I and $F$ liquid crystals where molecular tilts are normally accompanied by distortions of the hexagonal lattice in the direction of the tilt [26]. Possibly, this distortion exists in LB monolayers but is too small to detect in our experiment; however, an essential difference between the molecules of the smectics and those of the LB film may explain the lack of distortion in the monolayer case. In the smectic case, the molecules are effectively very densely packed cylinders. If molecules tilt, they press against the hard core repulsion of their neighbour in the direction of the tilt but not perpendicular to that direction. Thus, a hexagonal net of molecules distorts because of the tilt. In contrast, the alkyl chains in LB monolayer are kept slightly apart by the presence of the "head " group (carboxylate ion, cadmium ion, plus any solvation sphere). Therefore, tilting of molecules does not necessarily cause chains to press against the hard core repulsion of any neighbouring chain and no distortion will occur. Our present data cannot distinguish whether the molecular tilt directions in the monolayer are strictly related to the bond directions as the smectic I and F phases [26] or are decoupled from the underlying lattice as has been suggested for hexatic phases of tilted molecules [27].

Additional information on the molecular tilt in the monolayer can be obtained from the increasing radial FWHM with increasing $q_{z}$ found in our data. This phenomenon is incompatible with a layer which is translationally invariant in the $z$ direction and indicates a loss of in-plane correlations for momentum transfer vectors, $\mathbf{q}$, with increasing $q_{z}$ component. As was discussed earlier, the dense packing of hydrocarbon chains in the monolayer implies that the molecules are in all trans conformations thus eliminating one source of this loss of in-plane correlations. A structure containing coherently diffracting microdomains $\left(\ll 1 \mu \mathrm{m}^{2}-\right.$ the sampling area) each with different tilt directions but within which the tilt magnitude and direction are uniform is also incompatible with this feature of the observed diffraction pattern. Further, our modelling shows this feature cannot arise from the compositional anisotropy of the true cadmium stearate molecule making up the film. However, a likely model for this inhomogeneity along the $z$ direction in the monolayer is disorder in the tilt magnitude and/or direction of the molecules within a coherently diffracting cluster.
Motivated by an older model proposed by Langmuir [28] and Epstein [29] and by recent theoretical calculations [30], we have examined two potential models for tilt disorder in the monolayer. Both models arise because the monolayer structure tries to compensate for the difference between the equilibrium head-head and chain-chain distances each piece of the molecule would want to attain if it were independent. In one model, the magnitude of the tilt is fixed but the tilt direction wanders slowly through the lattice (see Fig. 5a). In the second model, the tilt always points to the centre of the cluster but the magnitude varies from straight up at the centre of the cluster to some maximum tilt at the cluster edge. Such a structure containing a splay distortion of the tail ordering is pictured in figure $5 \mathrm{~b}$ and will be called the « micellar

(A)
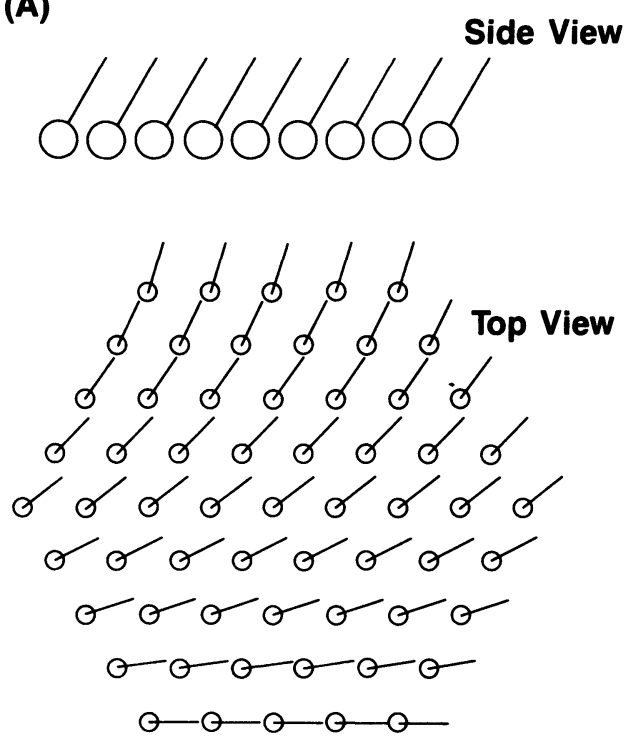

(B)
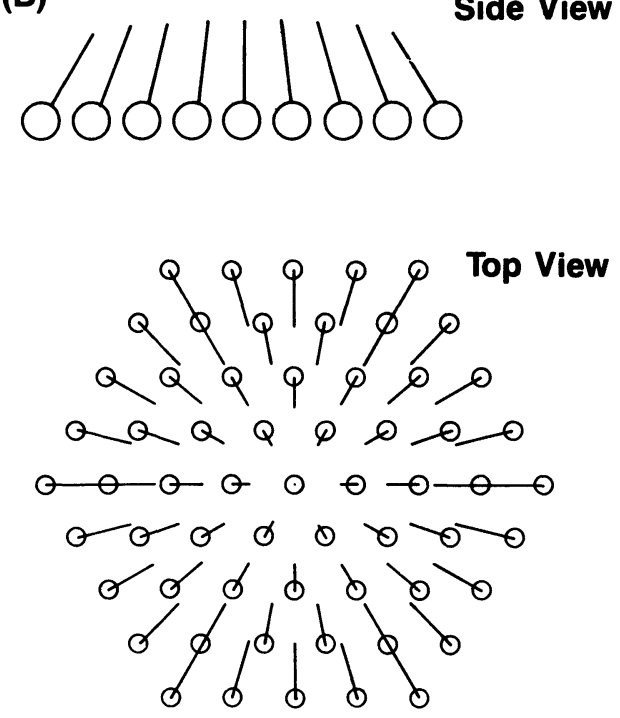

Fig. 5. - Schematics of tail directions in a cluster for : (a) model with slowly varying tilt direction; (b) micellar cluster model. 
cluster $»$. Both models exhibit contributions to translational disorder beyond that existing in the head group lattice. The micellar cluster model preserves strong bond orientational correlations while the first model degrades them to some extent. Our simulations show that the micellar model can fit the general features of our diffraction pattern better than the first model. Input parameters for the model include the tilt gradient across the cluster (which must be small because the small mismatch between the hard core size of the chain and the lattice constant prevents large tilts) and the size of the coherently diffracting unit (which must be small because of the short translational correlation length of the monolayer). Using reasonable values for these parameters $\left(\approx 25^{\circ}\right.$ variation of tilt angle across a cluster composed of $\approx 10^{2}$ molecules), the micellar model shows an increasing FWHM in $q_{\|}$ with increasing $q_{z}$ similar to our data. It can also simultaneously fit the FWHM of the radial and $q_{z}$ data while preserving narrow $\chi$ peaks. The fit of the micellar model to our $q_{z}$ data is shown in figure 4 . Increasing FWHM and decreasing intensity for the $\langle 110\rangle$ and $\langle 200\rangle$ spots at $q_{z}=0$ are also predicted by the micellar model.

The precise nature of the positional disorder, liquid-like or microcrystalline, is difficult to determine for the strongly disordered structure of complex molecules found in the LB monolayer. Essentially, one would like to know if the structure is similar to a stacked-hexatic phase (long range bond orientation with exponentially decaying translational correlations) [31] or microcrystalline (perfect translational correlations truncated by some set of defects which preserve bond-order, e.g. low-angle grain boundaries). The Lorentzian lineshape of the $\langle 100\rangle$ peak as well as the increasing FWHM and large decreases in intensity of higher index peaks are indicative that liquid-like correlations create the disorder in the monolayer. Further, the oval shape of the intensity contours are a signature of bond-oriented liquid-like structures in contrast to streaks found in the diffraction from bond-oriented microcrystalline structures [32]. Unlike for our monolayers, polycrystalline streaks are observed for unsupported LB lipid bilayers $[15,33]$ and large crystallites (on the order of $100 \mu \mathrm{m}$ ) have been observed in multilayer structures $[34,35]$.

The behaviour of the structure under the influence of the incident electron beam provides evidence that the poor translational order of the film is not beam induced. As noted earlier, the electron dose needed to record our diffraction patterns is less than those which show the onset of electron beam induced damage of LB films of lipids showing polycrystalline structures [15]. Our visual observations of the diffraction patterns are made with a further reduction of cumulative electron dose by an order of magnitude and show no qualitative differences from the recorded patterns. Particularly, the $\langle 100\rangle$ Bragg spots are not noticeably sharper radially or more streaked azimuthally during these immediate visual observations than in the recorded patterns. Thus, the poor translational and strong bond-oriented correlations seen in our films are not induced by electron beam damage.

Our observations of the behaviour of the film under large cumulative electron exposure indicate that the unperturbed monolayer structure measured in our diffraction experiment relaxes on the substrate subject to the lateral surface pressures and temperatures acting on the film. By chopping the electron beam, we have shown that this behaviour is, in fact, due to cumulative electron dose and is not a thermal effect due to local beam heating. Under much stronger illumination conditions than used in our measurements of the diffraction pattern, the spot pattern described rapidly disappears $(\approx 10 \mathrm{~s})$ and is replaced by an orthorhombic pattern of 6 very sharp spots (FWHM less than $1 / 20$ of the Bragg spots from the monolayer) with $d$-spacings of $4.2 \AA$ and $3.8 \AA$. The orientation of these spots is precisely aligned with the spots of the original monolayer. Imaging shows that the monolayer has ruptured and retracted, leaving behind small crystallites $(\approx 2 \mu \times 2 \mu$ basal plane area). The alignment of the crystalline spots with those of the original film suggests these crystallites form over sites where the film is more strongly pinned to the substrate. In contrast, prolonged exposure at the slow dose rates used to record diffraction patterns induces a gradual change in film structure. The Bragg spots slowly increase both radially (along $q_{\|}$) and azimuthally (along $\chi$ ), indicating a gradual loss of the last vestiges of translational order as well as degradation of the bond order. Concomittant with this loss of order, the lattice constant increases. When the translational correlation length is decreased to about $10 \AA$, the modulation of intensity along $\chi$ has become negligible, and the lattice constant has increased by roughly $5 \%$, the film ruptures and retracts across the substrate. The increased lattice constant is probably due to loss of molecules from the monolayer and thus decreased lateral surface pressure on the remaining molecules. Since this change in lattice constant occurs uniformly across virtually the entire film, the molecules must have sufficient mobility to adjust to this decrease in surface pressure. This surface pressure may be exerted by the pinning centres discussed earlier. Thus, it appears that at sufficient densities, the bondorientational order discussed earlier exists and the film can maintain a 2-dimensional, monolayer arrangement. However, at lower densities, this ordering is lost and the 2-dimensional arrangement becomes structurally unstable and ruptures. The loss of bond and translational order with increased lattice constant has also been observed in preliminary experiments probing the effect of higher temperature on the substrate and decreased surface pressure of the precursing film at the air/water interface. Thus, the structure of the LB film deduced from our diffraction 
pattern represents a state of the monolayer which stably adjusts to the lateral surface pressure and temperature imposed on the film.

\section{Summary and comments.}

Our electron diffraction study has revealed that our cadmium stearate LB monolayer has a stable, strong bond-orientation but poor translational order. With hexagonal symmetry, the monolayer is composed of unit cells containing a single hydrocarbon chain with significant rotational motion about its long axis. The bond-order of the monolayer is long range; the translational correlations - appearing to be liquidlike - exhibit a correlation length of about $40 \AA$. In many respects, its structure is similar to that of a stacked hexatic phase [31]. Dominantly in all trans conformations, hydrocarbon chains are tilted with respect to the layer normal, with the direction of the tilt equally probable at least at $60^{\circ}$ intervals within a $1 \mu^{2}$ area. Our diffraction pattern is entirely compatible with a structure proposed to arise from the mismatch in the natural head-head and chainchain separations which the components of the surfactant would seek if they were independent [28-30]. In this structure, molecules tilt in toward the centre of a 2-dimensional micelle-like cluster with increasing tilt magnitude from the centre to the periphery of the cluster. Such a model suggests that the molecular tilt and in-plane intermolecular order in these monolayers are much more decoupled then in smectic I and F phases. From changes in the film structure induced by the electron beam, it is clear that the monolayer is more strongly pinned to the substrate at certain, isolated positions and that the rest of the film (constrained to a 2-dimensional arrangement) relaxes under the surface pressure imposed by these pinning centres and the temperature of the film. At lower densities the film can no longer remain in the monolayer configuration and breaks up into crystallites.

Understanding the origins of the structure in this LB monolayer structure can shed light on the funda- mental free energy of surfactant structures in various environments. While there is considerable evidence that the preparation of the precursing film on the air/ water interface can affect molecular correlations in multilayer assemblies [34-36], the structure of the precursing monolayer is not yet known. If it is highly ordered, then the disorder of the monolayer on the substrate may be induced by strong uniaxial stresses in transferring the film to the solid substrate or by random field effects of the substrate potential on the monolayer [37]. Although recent second harmonic generation studies indicate that the molecular tilt may drive certain phase transitions at the air/water interface [38], the interplay between tilt and positional order in determining structures in these monolayers is yet to be completely understood. Unravelling this interplay is essential to understanding the fundamental free energies driving these monolayers as well as surfactant lamellar systems in general.

Speculations on the effects of the monolayer structure on its macroscopic properties are intriguing. Such poorly ordered structures may not be as effective as more ordered monolayers in serving as templates for subsequent layers in devices made of LB films [3]. In addition, a high degree of molecular ordering has been implicated in producing efficient boundary lubricants [2]; similarly a maximization of molecular density has been implicated in optimizing monolayers as dewetting agents [1]. Thus, the positional and tilt disorder found in these LB monolayers may inhibit their effectiveness in such macroscopic roles. If these types of disorder are endemic to the LB transfer process or hydrocarbon surfactants (where the head group of the surfactant is larger than the hydrocarbon chain), then their usefulness as boundary lubricants and dewetting agents may be in some question. The more open, splaying structure of these layers may also have effects on local chemical processes between the underlying surfaces and overlaying fluids. Thus, the hydrophobicity of these layers as well as their ability to protect surfaces from chemical attack may be inhibited by this splaying structure.

\section{References}

[1] Zisman, W. A., in Contact Angle : Wettability and Adhesion ed. Gould, R. F. (Advances in Chem. Series, ACS) 1964, Vol. 43, p. 1.

[2] BOWDEN, F. P. and TABOR, D., The Friction and Lubriction of Solids (Clarendon Press, Oxford) 1968, Part I, Chapter 10 and Part II, Chapter 18.

[3] See Proceedings of the First International Conference on Langmuir-Blodgett Films, in Thin Solid Films (1983) Vol. 99.

[4] Peterson, I. R., Russel, G. J., Philos. Mag. 49A (1984) 463.

[5] Bunnerot, A., Chollet, P. A., Frisby, H., Hoclet, A., Chem. Phys. 97 (1985) 365.
[6] Prakash, M., Dutta, P., Ketterson, J. B., AbrahaM, B. M., Chem. Phys. Lett. 111 (1984) 395.

[7] Chollet, P. A., Messier, J., Thin Solid Films 99 (1983) 197.

[8] Rabolt, J. F., Burns, F. C., Schlotter, N. E., Swalen, J. D., J. Chem. Phys. 78 (1983) 946.

[9] Germer, H. L., Storks, K. H., J. Chem. Phys. 6 (1938) 280.

[10] Chapman, J. A., Tabor, D., Proc. Roy. Soc. 242 (1957) 96.

[11] HuI, S. W., Biochim. Biophys. Acta 472 (1977) 345.

[12] Fischer, A., Lösche, M., Möhwald, H., SACKman, E., J. Physique Lett. 45 (1984) L-785. 
[13] Seul M., Eisenberger, P. and McConnell, H. M., Proc. Natl. Acad. Sci. USA 80 (1983) 5795.

[14] GAINes, G. L., Insoluble Monolayers at Liquid-Gas Interfaces (Wiley and Sons, Inc., New York) 1966, p. 187.

[15] HuI, S. W., Ultramicroscopy 5 (1980) 505.

[16] Dorset, D. L., Hui, S. W., Strozewski, C. M., J. Supermol. Struct. 5 (1976) 1.

[17] Glasser, R. M., Deamer, D. W., Proceedings of 27th EMSA Meeting (1969), 338.

[18] Hasmonay, H., Vincent, M., Dupeyrat, M., Thin Solid Films 68 (1980) 21.

[19] Edington, J. W., Practical Electron Microscopy in Material Science (Philips' Gloeilampenfabrieken, Einhoven) 1975, Appendix 8.

[20] Barraud, A., Rosilio, A., Le Gressus, C., Okuzumi, H., Mogami, A., Proc. 8th Intl. Congr. Electron Microscopy, vol. 1 (1974) 682.

[21] Garoff, S., Hall, R. B., De Santolo, A., Alvarez, M. S., to be published.

[22] Bothorel, P., Belle, J., Lamaire, B., Chem. Phys. Lipids 12 (1974) 96.

[23] Parsonage, N. G., Staveley, L. A. K., Disorder in Crystals (Clarendon Press, Oxford) 1978, p. 667680.

[24] Amoros, J. L., Molecular Crystals : Their Transforms and Diffuse Scattering (Wiley and Sons, New York) 1968, p. 56.
[25] Tilting of molecules in a monolayer sensitively depends on the hydrocarbon chain length, head group composition, and solid substrate. Thus, other monolayers of similar molecules show no tilt of the molecular axis (see Ref. [5]).

[26] Gane, P. A. C., Leadbetter, A. J. and Wrighton, P. G., Mol. Cryst. Liq. Cryst. 66 (1981) 247.

[27] Nelson, D. R. and Halperin, B. I., Phys. Rev. B 21 (1980) 5312.

[28] Langmuir, I., J. Chem. Phys. 1 (1933) 756.

[29] Epstein, H. T., Phys. Coll. Chem. 54 (1950) 1053.

[30] Safran, S. A., Robbins, M. O. and Garoff, S., to be published.

[31] Moncton, D. E. and Pindak, R., Phys. Rev. Lett. 43 (1979) 701.

[32] VaInshtein, B. K., Diffraction of X-Rays by Chain Molecules (Elsevier Pub., N.Y.) 1966, chap. 6.

[33] Hui, S. W., Parsons, D. F., Cowden, M., Proc. Nat. Acad. Sci, USA 71 (1974) 5068.

[34] Peterson, I. R., Thin Solid Films 116 (1984) 357.

[35] Veale, G., Peterson, I. R., J. Coll. Interf. Sci. 103 (1985) 178.

[36] Veale, G., Girling, I. R., Peterson, I. R., Thin Solid Films 127 (1985) 293.

[37] Sachdev, S. and Nelson, D. R., J. Phys. C 17 (1984) 5473.

[38] Rasing, Th., Shen, Y. R., Kim, M. W., Grubb, S., to be published. 